\title{
Pulmonary Embolism in Puerperium
}

\author{
Utsav Timalsina*, Rajan Sharma Kandel and Subarna Gautam
}

Department of Medicine, Rangeli Hospital, Rangeli, Morang, Nepal

\begin{abstract}
Pulmonary embolism is a sudden blockage in the lung artery. Pregnancy is an example of Virchow's triadhypercoagulability, stasis of blood and endothelial injury; together these factors lead to an increased incidence of venous thromboembolism.
\end{abstract}

Keywords: Pulmonary embolism; Puerperium; Pregnancy

\section{Introduction}

Pulmonary embolism is a sudden blockage in the lung artery. Pregnancy is an example of Virchow's triad- hypercoagulability, stasis of blood and endothelial injury; together these factors lead to an increased incidence of venous thromboembolism [1]. Most likely cause is deep vein thrombosis (DVT) in the leg or in the pelvis however in about $80 \%$ to $90 \%$ it occurs without any previous clinical manifestations of deep vein thrombosis [2] Clinical features depend upon the size of the embolus [2]. Pregnancy associated pulmonary embolism occurs suddenly and is not easy to diagnose promptly as the most common symptom of pregnancy associated pulmonary embolism is dyspnea which may be the presentation of normal pregnancy [3]. Death occurs from massive embolus within short time due to shock and vagal inhibition [2]. Pregnant women are fivefold at an increased risk of having venous thromboembolism than the non-pregnant women and the risk increases to $>20$ fold in the puerperium which remains increased until approximately 12 weeks postpartum [4,5]. Risk factors of Pregnancy associated pulmonary embolism are surgical history, preeclampsia, obesity, anemia, parity greater than 3 , age greater than 35 years and previous history of pregnancy associated pulmonary embolism [3]. Pregnancy related pulmonary embolism accounts for about $10 \%$ maternal deaths in the developed world [4]. Immediate diagnosis and prompt treatment is necessary for the improvement of pregnancy associated pulmonary embolism patient's survival rates [3].

\section{Case Report}

A 32-year-old, married, Hindu, Para 1 lady from Nepalgunj, Nepal working in service sector who had undergone emergency cesarean section 1 months back presented with chief complaints of Pain over the incision site for 21 days, discharge from the incision site for 21 days and fever for 17 days. She was apparently well 21 days back then she started having symptoms of wound infection, it was associated with fever, maximum temperature of $102^{\circ} \mathrm{F}$ associated with chills and rigor. There was no history of foul smelling per vaginal discharge. She developed sudden onset of shortness of breath with right sided chest pain on third day of admission. Patient was married for 2 years and she did not take any drugs. Emergency cesarean section was done for failed induction with outcome of single alive female baby weighing $3 \mathrm{~kg}$. She was a non-smoker and did not consume alcohol. On examination of patient during the third day of admission her general condition was ill looking, she was having difficulty in breathing, and pallor was present. Pulse rate was $100 / \mathrm{min}$ regular, respiratory rate was 26 / min, blood pressure was $100 / 60 \mathrm{mmHg}$, Spo 2 was $89 \%$ on room air; her axillary temperature was $98^{\circ} \mathrm{F}$. On examination of chest, air entry was bilaterally equal, normal vesicular breath sounds were heard with no added sounds. First and second heart sounds were heard with no murmurs. Abdominal examination revealed soft, non-tender uterus corresponding to 20 -week size, there was wound gap on the right edge of incision site. Renal angle tenderness was not present. There was no past history and family history of thrombophilias. On blood analysisHb: 9.9 gm\%, PCV: $32 \%$, WBC: $5890 / \mathrm{cmm}$, platelets: $371000 / \mathrm{cmm}$, RBS: $9.1 \mathrm{mmol} / \mathrm{L}$, urea: $5.8 \mathrm{mmol} / \mathrm{L}$ creatinine: $80 \mathrm{micro} \mathrm{mol} / \mathrm{L}$, sodium: $137 \mathrm{meq} / \mathrm{L}$, Potassium: $4.8 \mathrm{meq} / \mathrm{L}$, total bilirubin: $10 \mathrm{micromol} / \mathrm{l}$, direct bilirubin: 2 micromol/L, SGOT: 15.1 U/L, SGPT 16.7 U/L, ALP: 79 U/L, blood culture showed no growth, wound Swab culture showed no growth; Doppler ultrasonography of bilateral legs revealed normal venous doppler of bilateral legs; urinalysis was normal. There was sinus tachycardia in electrocardiogram. Chest X-ray PA view was normal, CT pulmonary angiogram revealed embolus in the right pulmonary artery. Patient was admitted in Medical intensive care unit (MICU), oxygen was given via face mask, injection piperacillin+tazobactum was started for wound infection [6] from the first day of admission, Injection Enoxaparin and Tab. Warfarin for pulmonary embolism was started from third day of her admission Patient showed improvement and was shifted to the obstetrics ward from MICU on her fourth day in MICU. Currently patient is in the obstetrics ward and her pulse rate is 80/min; Blood pressure: 110/70 mm Hg; respiratory rate: 15/min; SPO2: $95 \%$ without oxygen; wound is being regularly dressed under aseptic conditions; injection enoxaparin and tab warfarin is being continued (the consent of the patient has been obtained, in written form and verbally, for the presentation of this case study) (Figures 1-3).

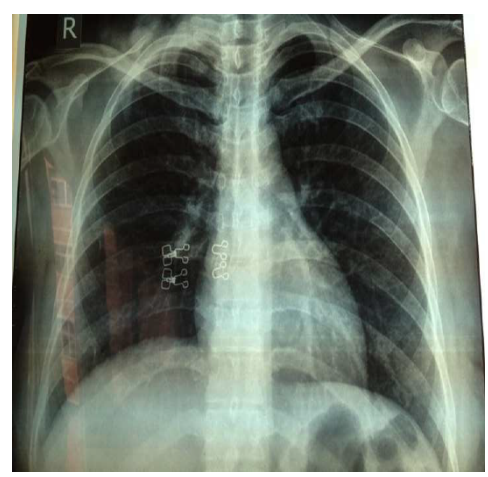

Figure 1: Normal chest X-ray PA view.

*Corresponding author: Utsav Timalsina, Department of Medicine, Rangeli Hospital, Rangeli, Morang, Nepal, Tel: +977-9851231711; E-mail: koolzhere@gmail.com

Received January 23, 2018; Accepted February 25, 2018; Published February 28,2018

Citation: Timalsina U, Kandel RS, Gautam S (2018) Pulmonary Embolism in Puerperium. J Clin Case Rep 8: 1084. doi: 10.4172/2165-7920.10001084

Copyright: @ 2018 Timalsina U, et al. This is an open-access article distributed under the terms of the Creative Commons Attribution License, which permits unrestricted use, distribution, and reproduction in any medium, provided the original author and source are credited. 


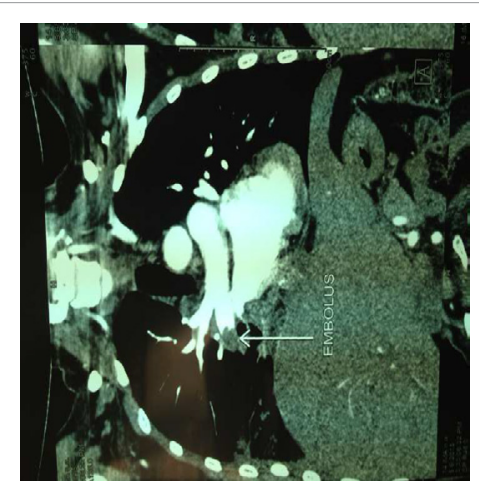

Figure 2: CT pulmonary angiogram showing embolus in the pulmonary artery.

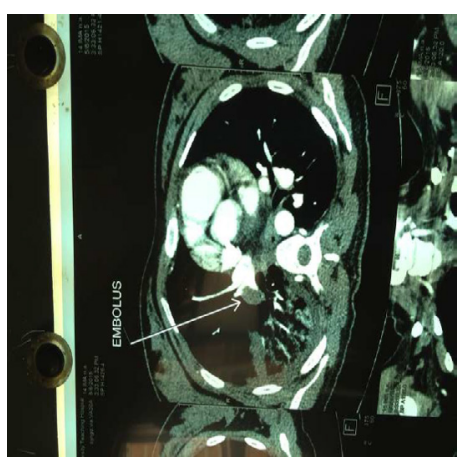

Figure 3: CT pulmonary angiogram showing embolus in the pulmonary artery.

\section{Discussion}

Pregnant women are fivefold at an increased risk of having venous thromboembolism than the non-pregnant women and the risk increases to $>20$ fold in the puerperium which remains increased until approximately 12 weeks postpartum [4,5]. The incidence of pulmonary embolism is three times greater in operative than in normal deliveries [7]. In about $80 \%$ to $90 \%$ it occurs without any previous clinical manifestations of deep vein thrombosis [2] as in this patient. In such women, ventilation perfusion scan or CT pulmonary angiogram should be performed [8]. In clinically suspected deep venous thrombosis or pulmonary embolism, treatment with low molecular weight heparin should begin immediately until the diagnosis is excluded [8]. Unfractionated heparin and warfarin are also acceptable in the postpartum period and the breast-feeding women [4]. The highest prevalence of pregnancy associated pulmonary embolism is after the cesarean section so patients after the cesarean section should be monitored more carefully than patients with vaginal delivery because surgery results in many hematological changes [3].

\section{Conclusion}

Mortality from pregnancy associated pulmonary embolism occurs within 30 minutes of inciting event which makes timely intervention more challenging [3]. Thus, it is very important for the obstetricians to recognize the risks of pulmonary embolism during pregnancy so that the woman with risk factors can be managed appropriately with thromboprophylaxis and mortality can be reduced [3].

\section{References}

1. Bourjeily G, Paidas M, Khalil H, Montella KR, Rodger M (2010) Pulmonary embolism in pregnancy. The Lancet 375: 500-512.

2. Konar H (2015) DC Dutta's Textbook of Obstetrics. Jaypee Brothers, Medical Publishers Pvt. Limited.

3. Lee MY, Kim MY, Han JY, Park JB, Lee KS (2014) Pregnancy-associated pulmonary embolism during the peripartum period: An 8-year experience at a single center. Obstetrics \& Gynecology Science 57: 260-265.

4. Marshall AL (2014) Diagnosis, Treatment, and Prevention of Venous Thromboembolism in Pregnancy. Postgraduate Medicine 126: 25-34.

5. Schmidt AP, Szeles TF, Santos WF, Auler JOC (2017) Massive pulmonary embolism during the postpartum period: Brief review about diagnosis, prophylaxis and early management. J Obstetrics Gynaecol 37: 1-4.

6. Wagner KJ, Bier U, Callies R, Regidor P, Schindler A (2006) Antibiotic prophylaxis in cesarean section - piperacillin versus piperacillin/tazobactam in 300 cesarean sections. Zentralbl Gynakol 128: 149-152.

7. Bunzel EE (1927) Pulmonary embolism complicating pregnancy, labor, and the puerperium. Am J Obstetrics Gynecol 13: 584-591.

8. The Royal College of Obstetricians and Gynaecologists (2015) Thromboembolic Disease in Pregnancy and the Puerperium: Acute Management. 3rd Edition pp $1-32$. 